\title{
Inhibition of NF-kappa B pathway leads to deregulation of epithelial-mesenchymal transition and neural invasion in pancreatic cancer
}

\author{
Alice Nomura ${ }^{1,2}, K_{\text {Kustav Majumder }}^{3}$, Bhuwan Giri ${ }^{1,2}$, Patricia Dauer ${ }^{1,4}$, Vikas Dudeja ${ }^{1,2}$, Sabita Roy ${ }^{1,2}$, \\ Sulagna Banerjee ${ }^{1,2}$ and Ashok K Saluja ${ }^{1,2}$
}

$\mathrm{NF}-\kappa \mathrm{B}$ has an essential role in the initiation and progression of pancreatic cancer and specifically mediates the induction of epithelial-mesenchymal transition and invasiveness. In this study, we demonstrate the importance of activated $\mathrm{NF}-\kappa \mathrm{B}$ signaling in EMT induction, lymphovascular metastasis, and neural invasion. Modulation of NF- $\kappa \mathrm{B}$ activity was accomplished through the specific NF-KB inhibitor (BAY 11-7085), triptolide, and Minnelide treatment, as well as overexpression of IKBa repressor and IKK activator plasmids. In the classical lymphovascular metastatic cascade, inhibition of NF-KB decreased the expression of several EMT transcription factors (SNAI1, SNAI2, and ZEB1) and mesenchymal markers (VIM and $\mathrm{CDH} 2$ ) and decreased in vitro invasion, which was rescued by IKK activation. This was further demonstrated in vivo via BAY 11-7085 treatment in a orthotopic model of pancreatic cancer. In vivo NF-KB inhibition decreased tumor volume; decreased tumor EMT gene expression, while restoring cell-cell junctions; and decreasing overall metastasis. Furthermore, we demonstrate the importance of active NF- $\mathrm{BB}$ signaling in neural invasion. Triptolide treatment inhibits Nerve Growth Factor (NGF) mediated, neural-tumor co-culture in vitro invasion, and dorsal root ganglia (DRG) neural outgrowth through a disruption in tumor-neural cross talk. In vivo, Minnelide treatment decreased neurotrophin expression, nerve density, and sciatic nerve invasion. Taken together, this study demonstrates the importance of NF-KB signaling in the progression of pancreatic cancer through the modulation of EMT induction, lymphovascular invasion, and neural invasion.

Laboratory Investigation (2016) 96, 1268-1278; doi:10.1038/labinvest.2016.109; published online 24 October 2016

Pancreatic cancer is now the third leading cause of cancer related death in the United States and is projected to be the 2nd by 2030 (ref. 1). The overall 5-year survival rate of this disease is a dismal $7 \%$, making it one of the very few cancers in single digits. ${ }^{2}$ The primary reasons for the poor prognosis is due to the late diagnosis, limited therapeutic options, early metastatic spread, and the aggressive nature of pancreatic cancer.

Tumor cell dissemination early in development has been demonstrated in pancreatic cancer and is considered to be one of the causes of recurrence even after surgical resection. ${ }^{3}$ The process of lymphovascular metastasis is a multistep cascade, in which tumor cells invade the basement membrane, intravasate into the vascular or lymphatic circulation, extravasate out of the circulation at a distant site, and develop into a metastatic lesion. The initiation of this process is orchestrated by the induction of the epithelial-mesenchymal transition (EMT), stimulating cells to lose cell-cell adhesions and thus, allowing tumor dissemination throughout the body.

In addition to the lymphovascular metastasis, the pancreatic tumor is also known to undergo neural invasion, in which the tumor uses the neural network for the spread of disease. ${ }^{4}$

Dissemination of tumor cells into the nerves, rather than the vascular or lymphatic circulation, has been shown in up to $100 \%$ of pancreatic cancer patients. ${ }^{5,6}$ Invasion into the nerves, or perineural invasion, is characterized as the invasion of tumor cells 'in, around, or through' the nerves. ${ }^{4}$ Neural invasion in pancreatic cancer has been contributed to the severe abdominal pain experienced by patients and tumor recurrence following curative surgical resection. ${ }^{4,7}$

'Division of Surgical Oncology, Department of Surgery Sylvester Comprehensive Cancer Center, University of Miami, Miami, FL, USA: ${ }^{2}$ Division of Basic and Translational Research, Department of Surgery, University of Minnesota, Minneapolis, MN, USA; ${ }^{3}$ Department of Surgery, University of Minnesota, Minneapolis, MN, USA and ${ }^{4}$ Department of Pharmacology, University of Minnesota, Minneapolis, MN, USA

Correspondence: Dr AK Saluja, PhD, Division of Surgical Oncology, Department of Surgery Sylvester Comprehensive Cancer Center, University of Miami, 1120 NW 14 th Street, Suite 610P, Miami, FL 33136, USA.

E-mail: asaluja@miami.edu

Received 2 August 2016; revised 7 September 2016; accepted 12 September 2016 
Contributing to the cross-talk between nerve and tumor, is the upregulation of neurotrophin and neurotrophin receptors in pancreatic cancer. Overexpression of nerve growth factor (NGF), brain derived neurotrophic factor (BDNF), and glial derived neurotrophic factor (GDNF) provide additional growth signals for nerves. Increased density of nerves and thickness of nerve bundles has been observed histologically in pancreatic ductal adenocarcinoma., ${ }^{7,8}$ In addition to the growth of nerves within the pancreatic tumor, these signals activate proliferative signaling pathways in the tumor and have been shown to increase invasiveness in pancreatic cancer cells. ${ }^{9-12}$

Induction of EMT and increased cellular invasiveness has been attributed to the induction of NF- $\kappa \mathrm{B}$ in many different cancer types, including pancreatic cancer. ${ }^{13,14} \mathrm{Up}$ to $70 \%$ of PDAC tumors show a constitutive activation of NF- $\kappa$ B and this has shown to be required for tumor development. ${ }^{15,16}$ Inhibition of this pathway may prove clinically effective in decreasing metastatic spread. Minnelide, a pro-drug derived from triptolide, has been shown in several cancers to downregulate NF- $\kappa$ B activity. ${ }^{17-19}$

In this study, we show the role of $\mathrm{NF}-\kappa \mathrm{B}$ in invasion and metastasis and that its inhibition using Minnelide treatment decreases EMT, invasion, and metastasis. We also examine the effect of Minnelide on neural invasion and show a disruption of neural-tumor cross talk via decreased expression of neurotrophin and neurotrophin receptors and neural invasion both in vitro as well as in vivo.

\section{MATERIALS AND METHODS}

\section{Cell Line Culture and Maintenance}

MIA PaCa-2 and AsPC-1 were obtained from ATCC. S2-VP10 was derived from the metastatic cell line, SUIT-2 (a gift from Professor Masato Yamamoto, University of Minnesota). KPC cell line was derived from LSL-Kras ${ }^{\mathrm{G} 12 \mathrm{D} /+}$; LSL-Trp53 ${ }^{\mathrm{R} 172 \mathrm{H} /{ }^{+}} ; \mathrm{Pdx}-1-\mathrm{Cre}$ transgenic mouse tumor as described by Banerjee et al,. ${ }^{20}$ Briefly, tumors were digested by collagenase $\mathrm{B}$ and dispase II and plated in medium (supplemented with growth factors and 2\% serum) for 2-3 weeks to remove fibroblasts. Established primary cell line was further grown in DMEM containing 10\% serum. Human pancreatic ductal epithelial cells were a gift from Professor Anil Rustgi and cultured as described in Reichert et al. ${ }^{21}$

MIA PaCa-2 and KPC cell line were grown in DMEM-high glucose, supplemented with 10\% fetal bovine serum; S2-VP10 cultured in RPMI with 10\% FBS; and AsPC-1 was cultured in RPMI with 20\% RPMI. All media was supplemented with 1X penicillin and streptomycin.

\section{Boyden Chamber Invasion Assay}

Invasion inserts (Corning BioCoat) were hydrate in serumfree medium; the bottom of the chamber contained the attractant $(10 \% \mathrm{FBS}$, recombinant NGF, or neural/cancer cells plated on the bottom of the well); 25,000 cells were plated on the top of the insert. At $24 \mathrm{~h}$, non-invaded cells were scrubbed from the top chamber via cotton swab, invaded cells were fixed in methanol, and stained by crystal violet. Invaded cells were counted by microscopy.

\section{Migration Assay}

Assay was conducted by Electric Cell-substrate Impedance Sensing (ECIS) (Applied Biophysics) and performed as described in Banerjee et al. ${ }^{20}$

\section{Immuohistochemistry}

Resected tissues were fixed in $10 \%$ formalin followed by $80 \%$ ethanol, paraffin embedded, sectioned, and deparaffinized in xylenes and hydrated through graded ethanol solutions. Antigen retrieval was performed in Reveal Decloaker (Biocare Medical) and background staining was minimized by blocking in Sniper Universal Blocking Sera (Biocare Medical). Slides were stained using anti-vimentin, anti-ncadherin, and anti-MMP9 antibodies (Cell Signaling) and incubated overnight. Alexefluor fluorescent antibody conjugates were used following primary antibody staining. Slides were counterstained by DAPI Prolong gold mounting medium (Life Technologies) and visualized by Leica fluorescent microscope. Negative controls were incubated with mouse IgG1 isotype control and did not demonstrate and specific staining.

\section{NF-KB Assay}

Activity of $\mathrm{NF}-\kappa \mathrm{B}$ was determined by p 50 binding ELISA (Thermo Scientific) performed according to manufacturer's protocol of whole cell lystates. Values were normalized to microgram protein, as measured by protein estimation (Thermo Scientific). Activation of NF- $\kappa \mathrm{B}$ was stimulated by $20 \mathrm{ng} / \mathrm{ml} \mathrm{TNF} \alpha$ (Sigma).

\section{NF-KB Modulation Plasmids}

Modulation of $\mathrm{NF}-\kappa \mathrm{B}$ signaling utilized transient plasmid overexpression by attractene transfection. Plasmids: IKK (IKK-2 S177E, S191E) and IKB $\alpha$ (pBabe-Puro-IKBalphamut(superrepressor)) plasmids (Addgene).

\section{Animals Models}

All experiments were performed according to the University of Minnesota Animal Care and Use Committee guidelines.

\section{KPC Murine Model}

${ }_{\text {LSL-Kras }}{ }^{\mathrm{G} 12 \mathrm{D} /+} ; \mathrm{LSL}-\mathrm{Trp} 53^{\mathrm{R} 172 \mathrm{H} /+} ; \mathrm{Pdx}-1-\mathrm{Cre}$ transgenic mice were treated with $0.42 \mathrm{mg} / \mathrm{kg}$ body weight per day Minnelide starting at 4-6 weeks until moribund. Tumor tissues were utilized for primary tumor cell line establishment, immunohistochemistry for nerve staining (PGP9.5), and gene expression levels via quantitative PCR.

\section{PDX Subcutaneous Model}

Deidentified clinical pancreatic adenocarcinoma tumor samples (PDX1 and PDX2) were obtained from the University of Minnesota Department of Surgery. Samples were implanted 
subcutaneously into the flank of NOD/SCID mice. When tumors measured approximately $700-800 \mathrm{~mm}^{3}$, they were removed; cut into approximately $1-2 \mathrm{~mm}$ pieces; and implanted subcutaneously into the right flank of age matched NOD/SCID mice. Propagated tumors were used for experiments. PDX1 and PDX2 are two patient derived xenografts. When tumors measured $750 \mathrm{~mm}^{3}, 0.42 \mathrm{mg} / \mathrm{kg}$ Minnelide treatment was started. Animals were sacrificed at 10 days and tumor was harvested.

\section{Orthotopic Animal Model}

One million MIA PaCa-2 cells suspended in 1:1 PBS/ Matrigel were injected into the tail of the pancreas of athymic nude female mice via Hamilton syringe. At 2 weeks, $7.5 \mathrm{mg} / \mathrm{kg}$ BAY 11-7085 was administered IP every other day for 3 weeks. Control animals received DMSO.

\section{Sciatic Nerve Invasion Assay}

Fifty thousand MIA PaCa-2 cells were injected into the right sciatic nerve using a dissecting microscope and 33 gauge needle in $3 \mu$ l. Minnelide treatment started at day 7 after cell injection. Mice were observed for 6 weeks for gross behavior, limb function, and sciatic nerve function, as determined by paw spread distance.

\section{DRG Outgrowth Assay}

Dorsal root ganglia were isolated from the lower lumbar section of an adult mouse and placed in a droplet of Matrigel (Corning) in a 24-well plate. DRG/Matrigel were maintained in DMEM/F12 medium supplemented with 10\% FBS. Conditioned medium was removed from KPC cells cultured for $48 \mathrm{~h}$ and diluted 1:1 in with fresh medium. Dorsal root ganglia (DRGs) were imaged via microscope daily.

\section{Statistical Methods}

Values are expressed as mean \pm s.e.m. In vitro experiments were all independently performed at least three times. Statistical significance was calculated by Student's test or ANOVA analysis and significance $\left.{ }^{*}\right)$ is defined as $P<0.05$.

\section{RESULTS \\ NF-KB is Activated in Pancreatic Cancer and Imparts Invasiveness}

$\mathrm{NF}-\kappa \mathrm{B}$ pathway is a major pro-proliferative pathway in a number of cancers including pancreatic cancer. ${ }^{16}$ Our results show that the NF- $\kappa \mathrm{B}$ pathway is constitutively activated in pancreatic cancer compared with the normal ductal cells. NF$\kappa \mathrm{B}$ activity is significantly higher in several established pancreatic cancer cell lines AsPC-1 (16.31-fold \pm 2.70$)$; MIA PaCa-2 (4.08-fold \pm 0.43$)$; and S2-VP10 (15.26-fold \pm 2.87$)$ and tumors KPC1 (8.43-fold \pm 1.16 ); KPC2 (8.56-fold \pm 2.32); PDX1 (5.22-fold \pm 1.89$)$; and PDX2 (8.06-fold \pm 2.78 ) (Figure 1a). Along with regulating proliferation of tumor cells, NF- $\kappa$ B pathway also has a significant role in regulating the EMT as well as invasion in pancreatic cancer. ${ }^{22-24}$
In addition, our results also show that treatment with Minnelide results in significant downregulation of NF- $\kappa$ B activity both in vitro (Figures $\mathrm{lb}$ and $\mathrm{c}$ ) as well as in vivo (Figure 1d). Downregulation of NF- $\kappa \mathrm{B}$ also results in the downregulation of key EMT players (Figure 1e) as well as key genes involved in tumor-neural cross-talk (Figure 1f).

$\mathrm{NF}-\kappa \mathrm{B}$ inhibition also resulted in decreased EMT gene expression (Figure 1e). Treatment by BAY 11-7085 for inhibition of NF- $\kappa \mathrm{B}$ signaling decreased several EMT genes: SNAI1 (0.507-fold \pm 0.146$), \quad$ SNAI2 $\quad(0.357$-fold \pm 0.161$)$, ZEB1 (0.584-fold \pm 0.139$)$, VIM (0.322-fold \pm 0.022$)$, and $\mathrm{CDH} 2$ (0.495-fold \pm 0.259$)$. BAY 11-7085 treatment also decreased neurotrophin gene expression: ARTN (0.534fold \pm 0.097$)$, GDNF (0.390-fold \pm 0.103$)$, and NGF (0.139fold \pm 0.069$)$ as compared with untreated samples.

\section{NF-kB Activity is Required For Pancreatic Cancer Invasiveness}

Next we wanted to determine if inhibition of NF- $\kappa \mathrm{B}$ signaling does indeed decrease cellular invasiveness in pancreatic cancer. Through inhibition of NF- $\kappa \mathrm{B}$ activity by $\operatorname{IKB} \alpha$ repressor plasmid and BAY 11-7085 pharmacological inhibition we saw a decrease in Boyden chamber invasion as compared with untreated control (Figure 2a). IKB $\alpha$ repressor plasmid expression decreased invasion to $0.46( \pm 0.025)$ of control and BAY 11-7085 treatment decreased invasion to $0.02( \pm 0.008)$ of untreated control. Pharmacological inhibition via BAY 11-7085 also decreased EMT marker, vimentin, at the protein level (Figure $2 b$ ).

Next we wanted to confirm that NF- $\kappa \mathrm{B}$ was responsible for the effect of triptolide on EMT and invasion. Triptolide treatment decreased NF- $\kappa$ B activity to 0.66 -fold $( \pm 0.089)$ of untreated MIA PaCa-2 control. Cells expressing the IKK enhancer plasmid treated with triptolide more than restored the NF- $\kappa \mathrm{B}$ activity that triptolide diminished (4.104fold \pm 0.701 ; Figure $2 \mathrm{c}$ ).

To determine whether NF- $\kappa \mathrm{B}$ signaling is mediating the downregulation of EMT gene expression from triptolide treatment, we expressed the IKK enhancer plasmid to rescue the effects of triptolide treatment. Triptolide treatment decreased SNAI1 $\quad(0.693$-fold \pm 0.270$)$, SNAI2 $(0.259$-fold \pm 0.048$)$, TWIST1 (0.205-fold \pm 0.080$)$, ZEB1 (0.407-fold \pm 0.187$)$, VIM $(0.589$-fold \pm 0.160$)$, and $\mathrm{CDH} 2(0.695$-fold \pm 0.055$)$ gene expression as compared with untreated control. With the expression of the IKK enhancer in addition to triptolide treatment, expression of these genes is rescued to approximately untreated levels or higher: SNAI1 (1.650-fold \pm 0.095$)$, SNAI2 (1.135-fold \pm 0.154$)$, TWIST1 $(4.099$-fold \pm 0.367$)$, ZEB1 (0.900-fold \pm 0.364$)$, VIM (3.554-fold \pm 0.607$)$, and $\mathrm{CDH} 2$ (1.357 fold \pm 0.181$)$, as compared with untreated control (Figure 2d).

We then wanted to determine whether this rescue was functionally significant in the context of invasion. Control, triptolide treated, and IKK enhancer expressing triptolide treated cells were examined for invasive potential. Triptolide 
decreased Boyden chamber invasion (0.0282-fold \pm 0.009$)$ and cells expressing the IKK enhancer plasmid treated with triptolide rescues this effect $(0.617$-fold \pm 0.080$)$ (Figure 2e).
These data together demonstrate that decreased NF- $\kappa$ B activity through plasmid, pharmacological inhibition, or triptolide treatment downregulates EMT genes and decreases invasiveness.

a
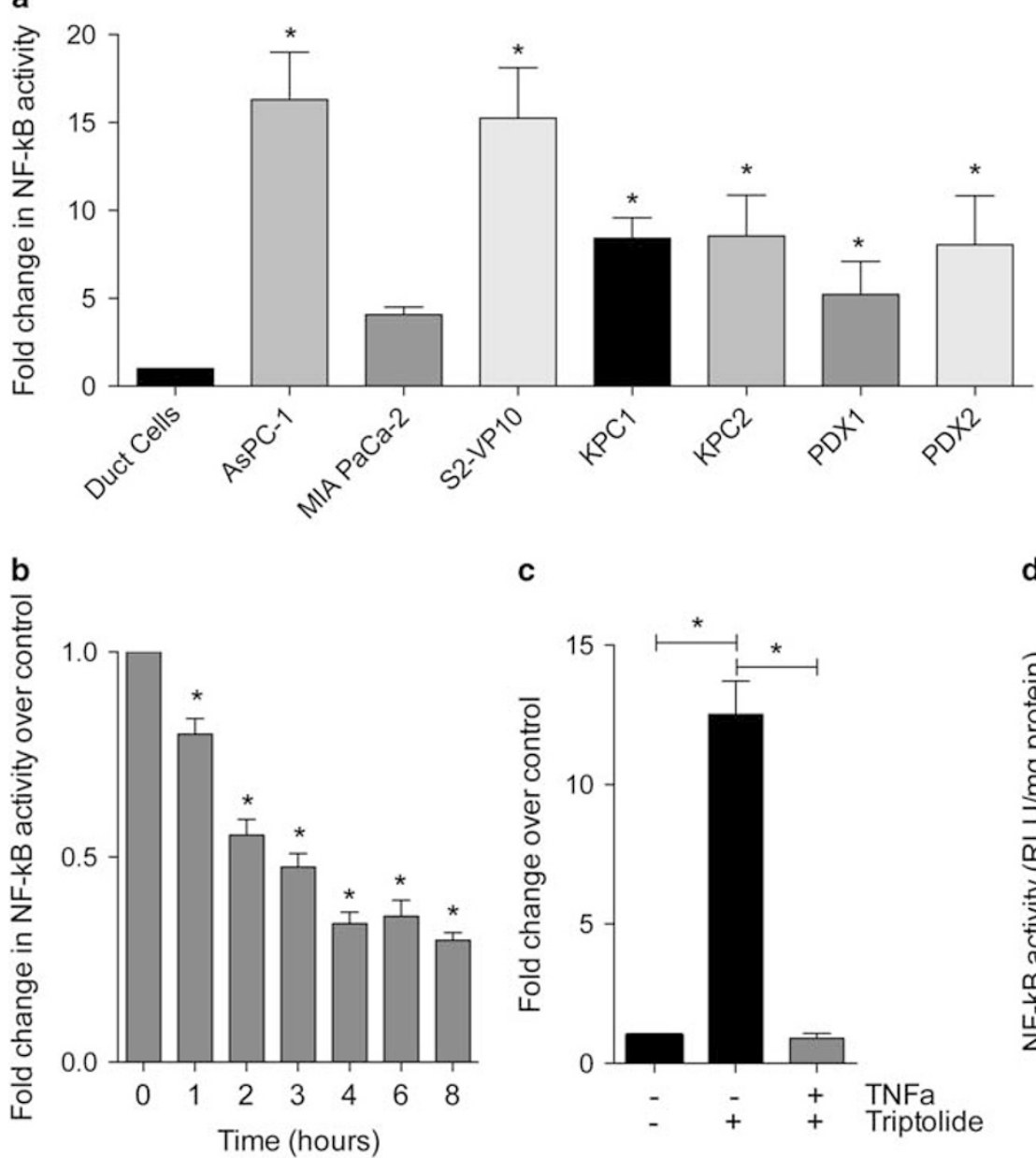

c

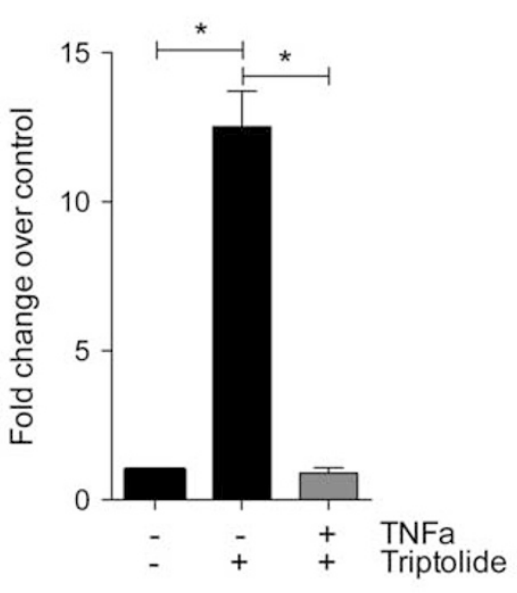

d

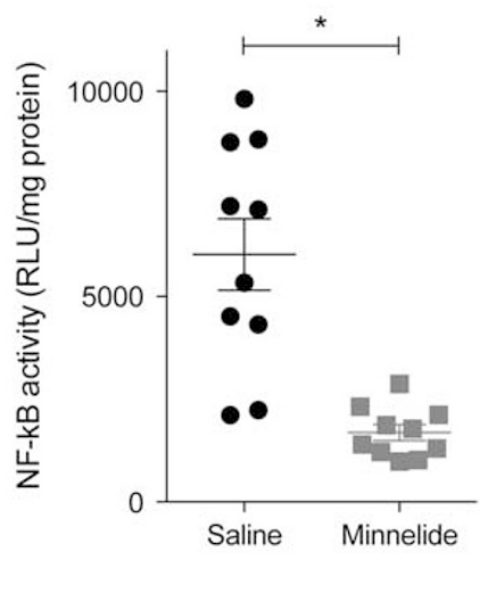

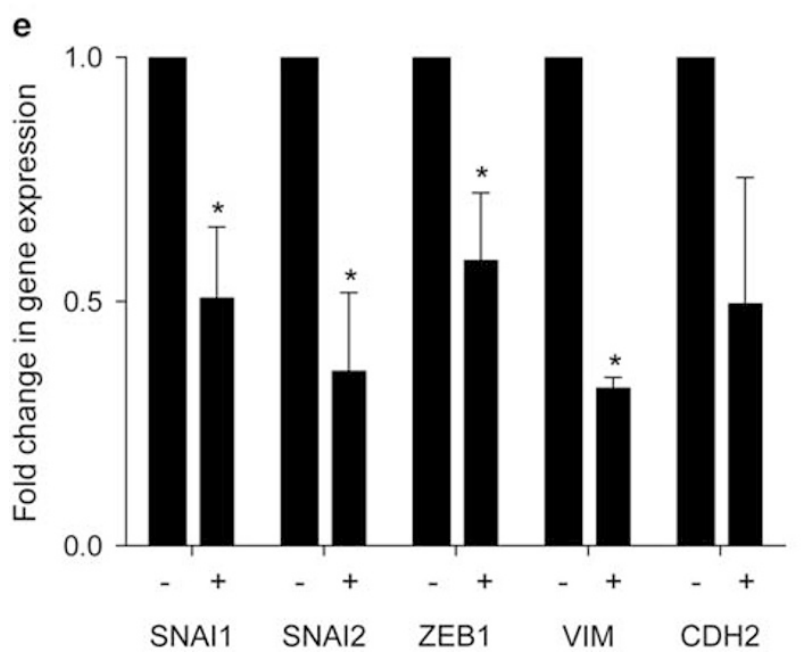

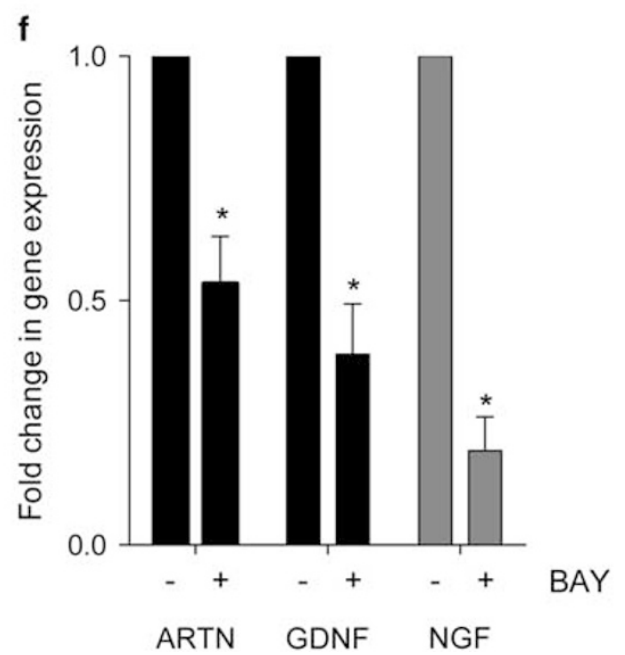

Figure $1 \mathrm{NF}-\kappa \mathrm{B}$ is activated in pancreatic cancer and imparts invasiveness: (a) increased p50-binding activity in several cell lines and tumors, as compared with cell lines; (b) triptolide treatment inhibition of NF- $\kappa B$ activity in a time dependent manner; (c) triptolide inhibits TNF $a$-induced NF- $\kappa B$ activity; (d) decreased NF-KB activity in vivo in MIA PaCa-2 tumors treated with Minnelide; (e) BAY 11-7085 treatment decreased (e) EMT gene expression and (f) neurotrophin gene expression in S2-VP10 cell line. Each bar is representative of three or more independent experiments; error bars are represented in s.e.m.; and the asterisk $\left(^{*}\right)$ indicates a $P$-value $<0.05$. 
a

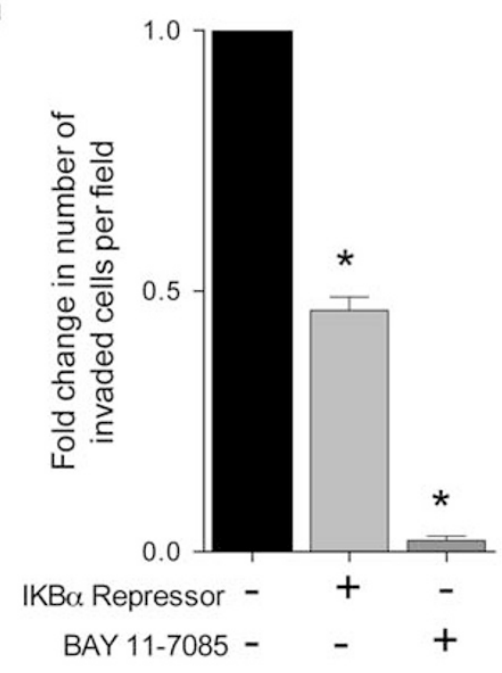

b

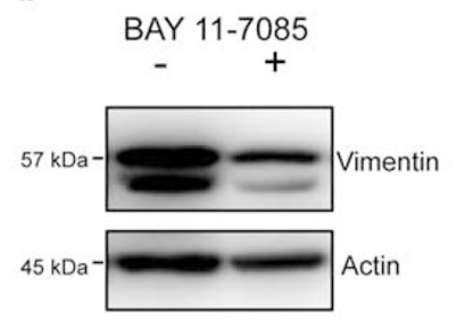

c

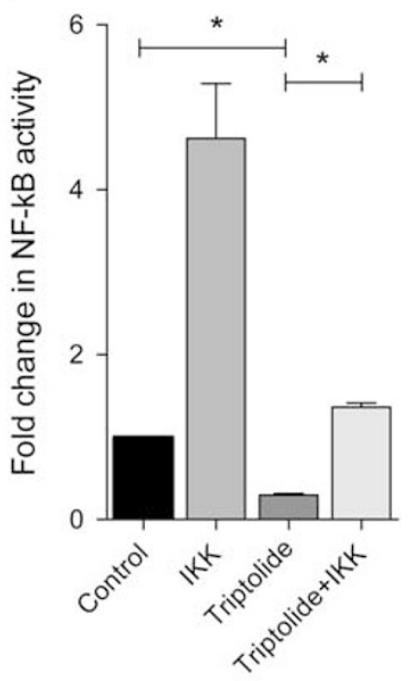

d

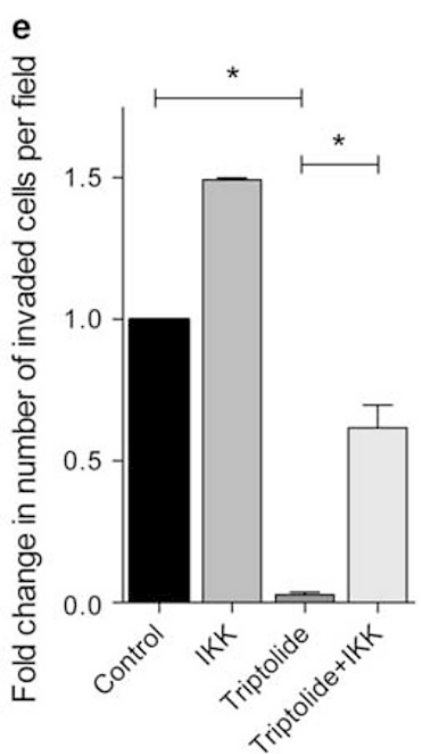

Figure 2 NF- $\kappa$ B activity is required for invasion: (a) Inhibition of NF- $\kappa$ B through IKBa repressor plasmid expression or BAY $11-7085$ treatment decreased cellular invasiveness via Boyden chamber invasion assay; (b) BAY 11-7085 treatment decreased vimentin protein expression; expression of IKK plasmid rescues triptolide inhibition of (c) NF- $\kappa$ B activity; (d) EMT gene expression; (e) Boyden chamber invasion. Each bar is representative of three or more independent experiments; error bars are represented in s.e.m.; and the asterisk $\left(^{*}\right)$ indicates a $P$-value $<0.05$.

\section{Inhibition of EMT in PDAC Decreases Invasion Potential and Metastatic Spread}

Early gene expression changes with Minnelide treatment in a human tumor xenograft model were assessed 7 days after start of treatment. EMT-associated genes, upregulated during this transition were decreased significantly with Minnelide treatment. EMT inducing transcription factors: SNAI1 (0.503 \pm 0.070 -fold), SNAI2 (0.408 \pm 0.061 -fold), and ZEB1 $(0.350 \pm 0.060$-fold $)$ were significantly down regulated. MMP9, VIM, and $\mathrm{CDH} 2$ decreased to $0.227 \pm 0.063$, $0.569 \pm 0.061$, and $0.539 \pm 0.056$ of saline control, respectively (Figure 3a).
In addition to the downregulation of EMT associated genes with Minnelide treatment, this effect was also seen at the protein level. Tumor sections from saline or Minnelide treated tumors demonstrated a downregulation of vimentin, MMP9, and N-cadherin via immunofluorescence (Figure 3b).

Inhibition of NF- $\kappa \mathrm{B}$ through Minnelide treatment in vivo is also demonstrated in the transgenic, spontaneous KPC murine model. EMT related transcription factor gene expression upon Minnelide treatment decreased in SNAI2 $(0.511 \pm 0.234$-fold $)$, and ZEB1 (2.075e $-5 \pm 6.853 e-6$-fold) as compared with saline tumors. Mesenchymal markers VIM, CDH2, MMP7, and MMP9 decreased to $0.048 \pm 0.018,0.655 \pm 0.137,0.146 \pm 0.096$, and 
a

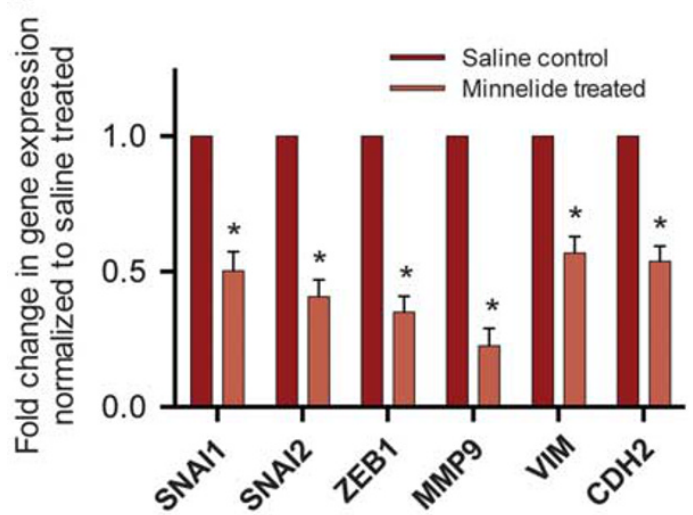

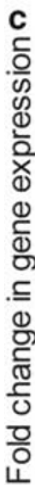

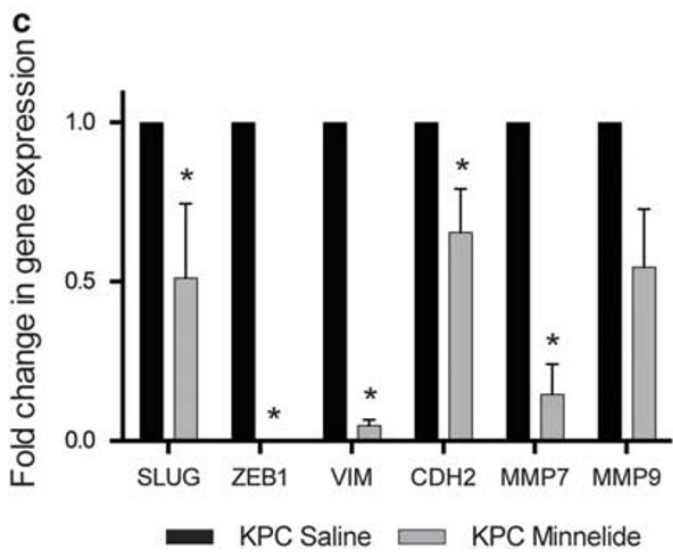

b

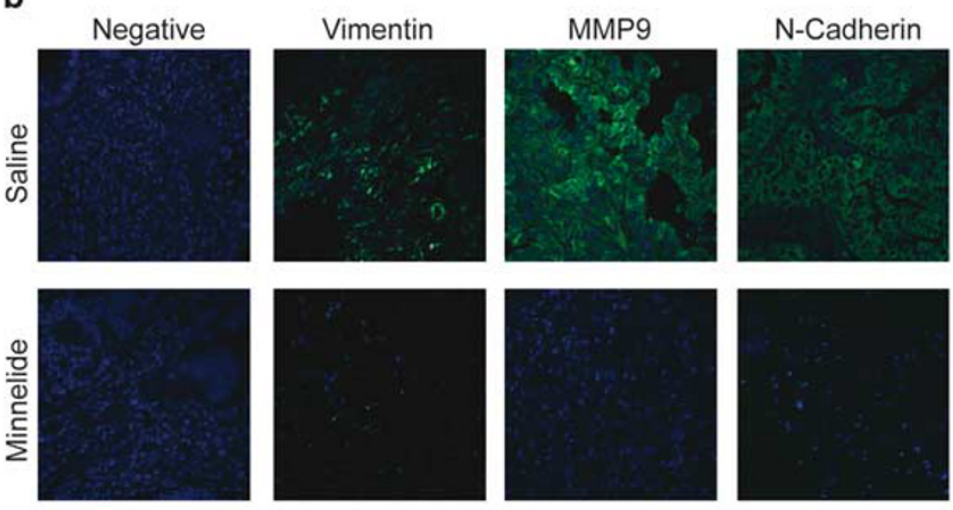

d

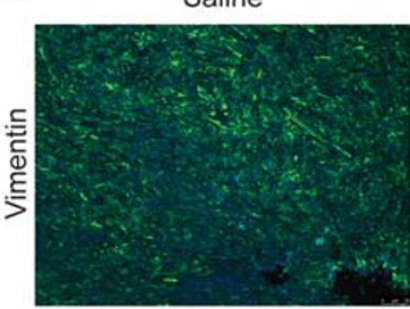

Minnelide

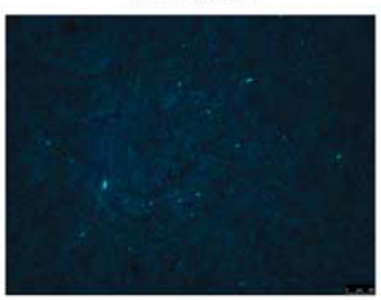

Figure 3 Inhibition of NF- $K B$ in PDAC decreases EMT related marker expression in vivo: Minnelide treatment in patient derived xenografts decreased (a) EMT gene expression and (b) mesenchymal marker expression as well as in KPC tumors $\mathbf{c}$ and $\mathbf{d}$, respectively. Each bar is representative of three or more independent experiments; error bars are represented in s.e.m.; and the asterisk $\left(^{*}\right)$ indicates a $P$-value $<0.05$.

$0.545 \pm 0.183$ of saline tumor control, respectively (Figure 3c). Vimentin expression is also markedly decreased by Minnelide treatment as seen via immunofluorescence (Figure 3d).

Triptolide also significantly decreased in vitro migration (Supplementary figure 1a) and invasion (Supplementary Figure 1b) in KPC cell line, as well as, invasion in established human pancreatic ductal adenocarcinoma cell lines. Invasion as compared with untreated control decreased by $97.7 \%$ and 97.4\% in MIA PaCa-2 and SUIT-2 derived S2013, respectively (Supplementary Figure 1c).

On the basis of these data we concluded that Minnelide inhibits EMT, limiting cellular motility, and invasiveness. We have previously demonstrated that in vivo Minnelide treatment markedly decreases metastasis to distant sites in a murine model of pancreatic cancer, ${ }^{25}$ these results demonstrate that this is likely due to $\mathrm{NF}-\kappa \mathrm{B}$ inhibition decreasing EMT and cellular invasiveness.

\section{Inhibition of NF-кB inhibits Epithelial-Mesenchymal Transition}

To determine whether the EMT inhibition and invasion which was seen in vitro translates in vivo, we used BAY
11-7085 treatment via intraperitoneal injection to inhibit $\mathrm{NF}-\kappa \mathrm{B}$ signaling in an orthotopic mouse model of pancreatic cancer. Treatment for 3 weeks resulted in a decrease in primary tumor weight $(0.561 \mathrm{~g} \pm 0.078$ in the control group versus $0.250 \mathrm{~g} \pm 0.052$ in the BAY 11-7085 treatment group; Figure $4 \mathrm{a})$. Tumor NF- $\kappa \mathrm{B}$ activity was determined by $\mathrm{p} 50$ binding ELISA, in which BAY 11-7085 treated tumors displayed decreased p50 binding (9657 RLU/ $\mu$ g protein \pm 586.6) as compared with DMSO control (7248 RLU/ $\mu \mathrm{g}$ protein \pm 538.3 ) (Figure 4b). BAY 11-7085 treated tumors also exhibited decreased gene expression of EMT transcription factors (SNAI2 0.745 fold \pm 0.070 and TWIST1 0.737 -fold \pm 0.164 ) and mesenchymal marker (N-Cadherin 0.377 -fold \pm 0.196$)$; increased E-cadherin expression (2.392fold \pm 0.265$)$; and decreased MMP7 gene expression (0.002fold \pm 0.001 ) (Figure 4c).

When observing the spread of disease in these mice, after 3 weeks there was metastasis to several distant sites within the DMSO control group, with a lower extent of metastasis in the BAY 11-7085 group (Figure 4d). Representative images of the abdominal cavity are shown in Figure $4 \mathrm{e}$, with metastatic lesions circled in white. In addition, these tumors demonstrate 
an increased E-cadherin staining at the cell membrane, indicating an increase in cell-cell adhesion (Figure 4f).

Together, these data demonstrate that invasion in vitro and metastasis in vivo are mediated by NF- $\kappa \mathrm{B}$ signaling and inhibition of this pathway inhibits EMT.

\section{Inhibition of Neurotrophic Signaling Decreases Neural Invasion in Pancreatic Cancer}

As described in Figure 1, NF- $\kappa \mathrm{B}$ inhibition decreases neurotrophin gene expression in pancreatic cancer cells. Using triptolide as a NF- $\kappa \mathrm{B}$ inhibitor, we wanted to study the
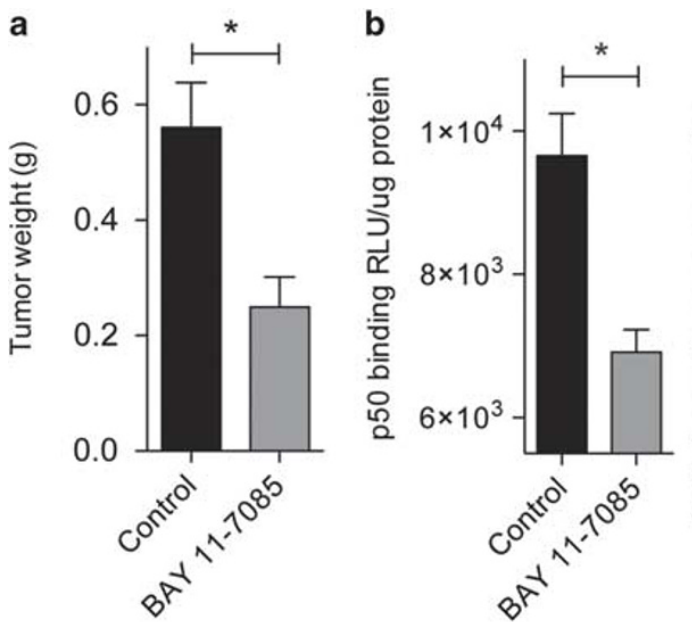

d

\begin{tabular}{|c|cc|} 
& DMSO Control & BAY $(7.5 \mathrm{mg} / \mathrm{kg})$ \\
\hline Spleen & $5 / 6$ & $2 / 6$ \\
Liver & $2 / 6$ & $0 / 6$ \\
\cline { 2 - 3 } Lymph nodes & $4 / 6$ & $0 / 6$ \\
Diaphragm & $2 / 6$ & $0 / 6$ \\
Abdominal Wall & $2 / 6$ & $0 / 6$
\end{tabular}

e
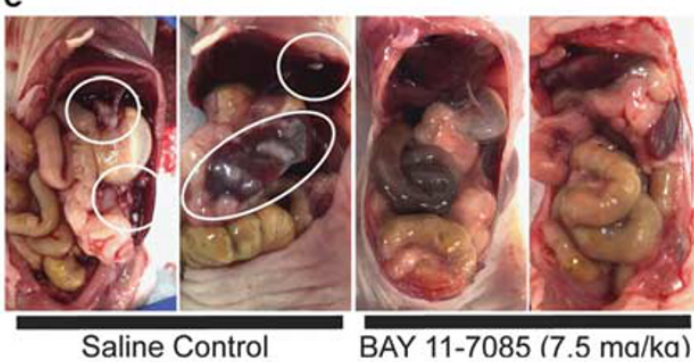

f

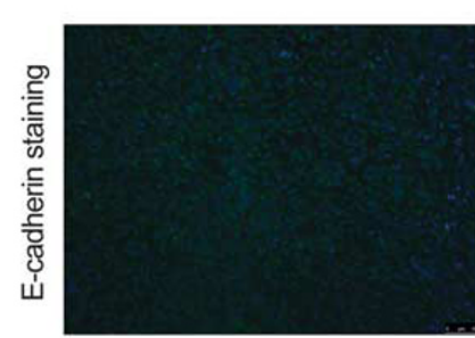

Control

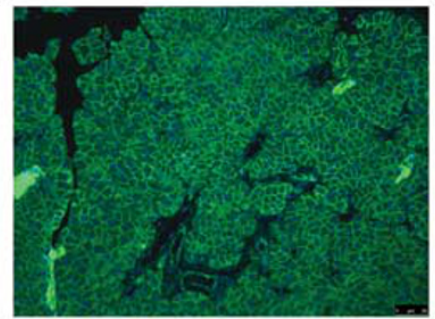

BAY $(7.5 \mathrm{mg} / \mathrm{kg})$

Figure 4 Inhibition of NF-KB inhibits EMT and metastasis in vivo: BAY 11-7085 treatment in a orthotopic MIA PaCa-2 model decreases (a) tumor weight; (b) tumor NF-KB activity; (c) tumor EMT gene expression; and (d) (e) metastasis to distant sites. Each bar is representative of three or more independent experiments; error bars are represented in s.e.m.; and the asterisk $(*)$ indicates a $P$ value $<0.05$.

Figure 5 Inhibition of neurotrophic signaling decreases neural invasion in pancreatic cancer cells: triptolide treatment inhibits increased MIA PaCa-2 invasion mediated by (a) NGF stimulation and (b) neural co-culture; (c) triptolide also inhibits SHSY-5Y invasion stimulated by MIA PaCa-2 co-culture; (d) SHSY-5Y co-culture increases MIA PaCa-2 neurotrophin and EMT-related gene expression; (e) conditioned medium from KPC cells increased neurite outgrowth from murine DRG and triptolide treatment decreases this outgrowth; (f) triptolide treatment decreased neurotrophin and neurotrophin receptor gene expression in cultured murine DRGs. Each bar is representative of three or more independent experiments; error bars are represented in s.e.m.; and the asterisk $\left(^{*}\right)$ indicates a $P$-value $<0.05$. 
a

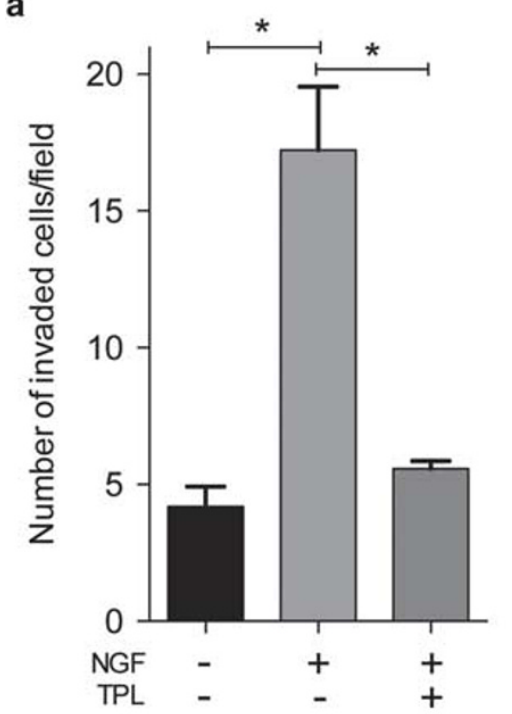

b

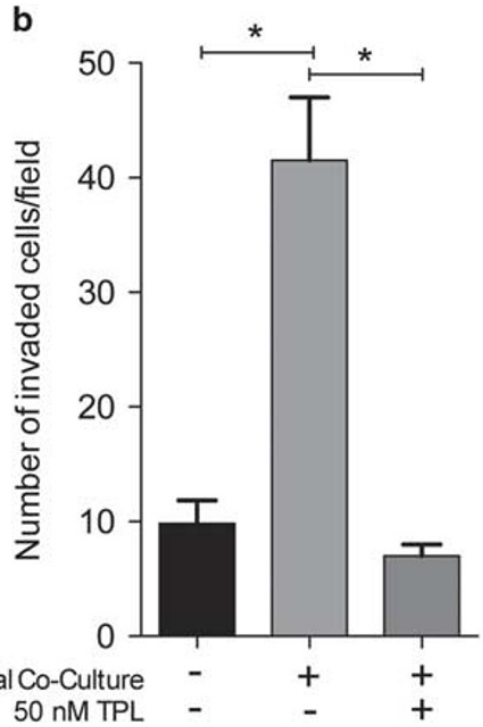

c

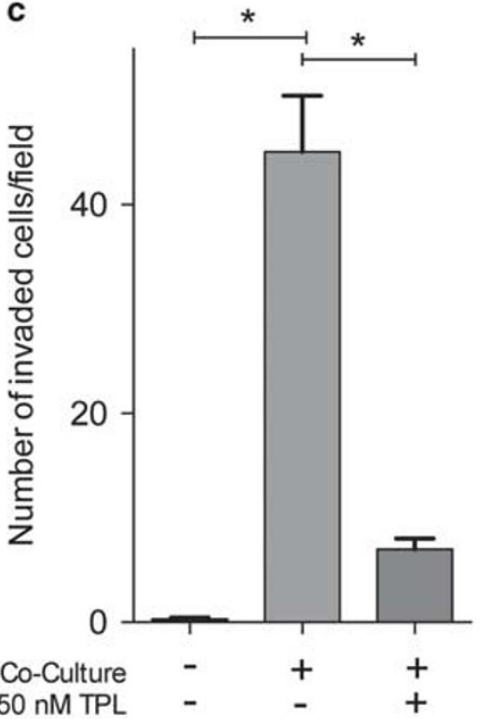

d
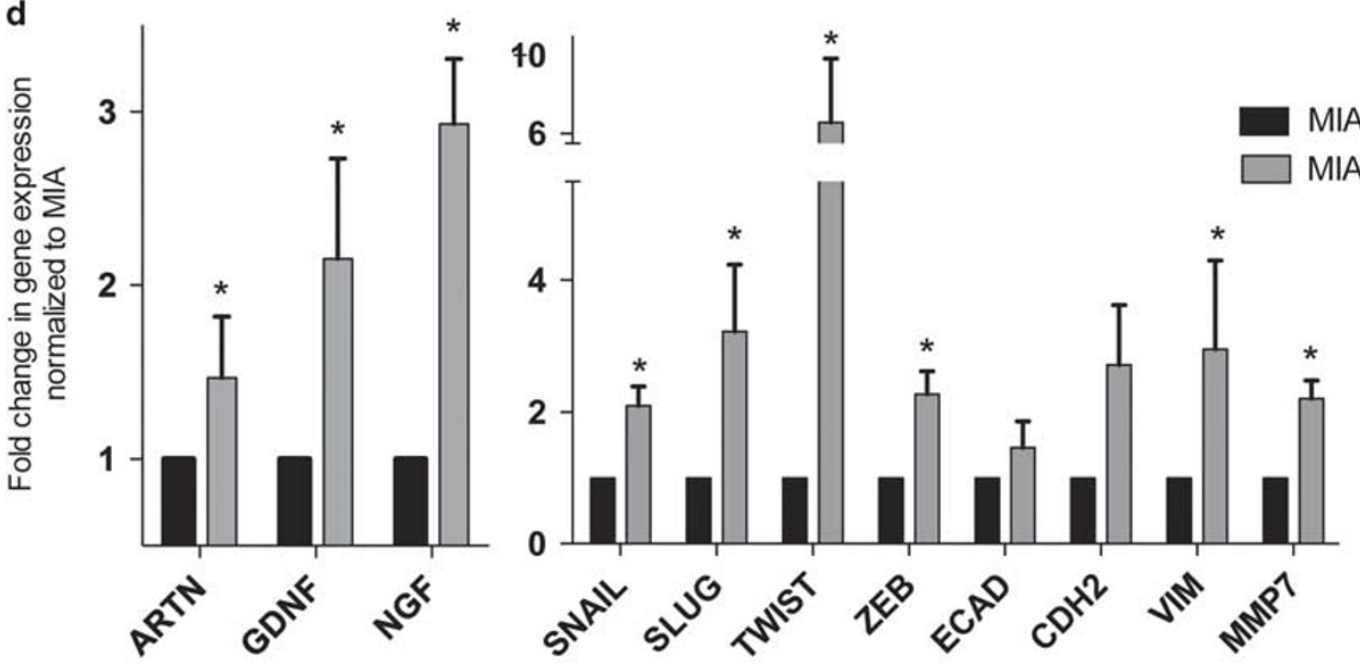

e
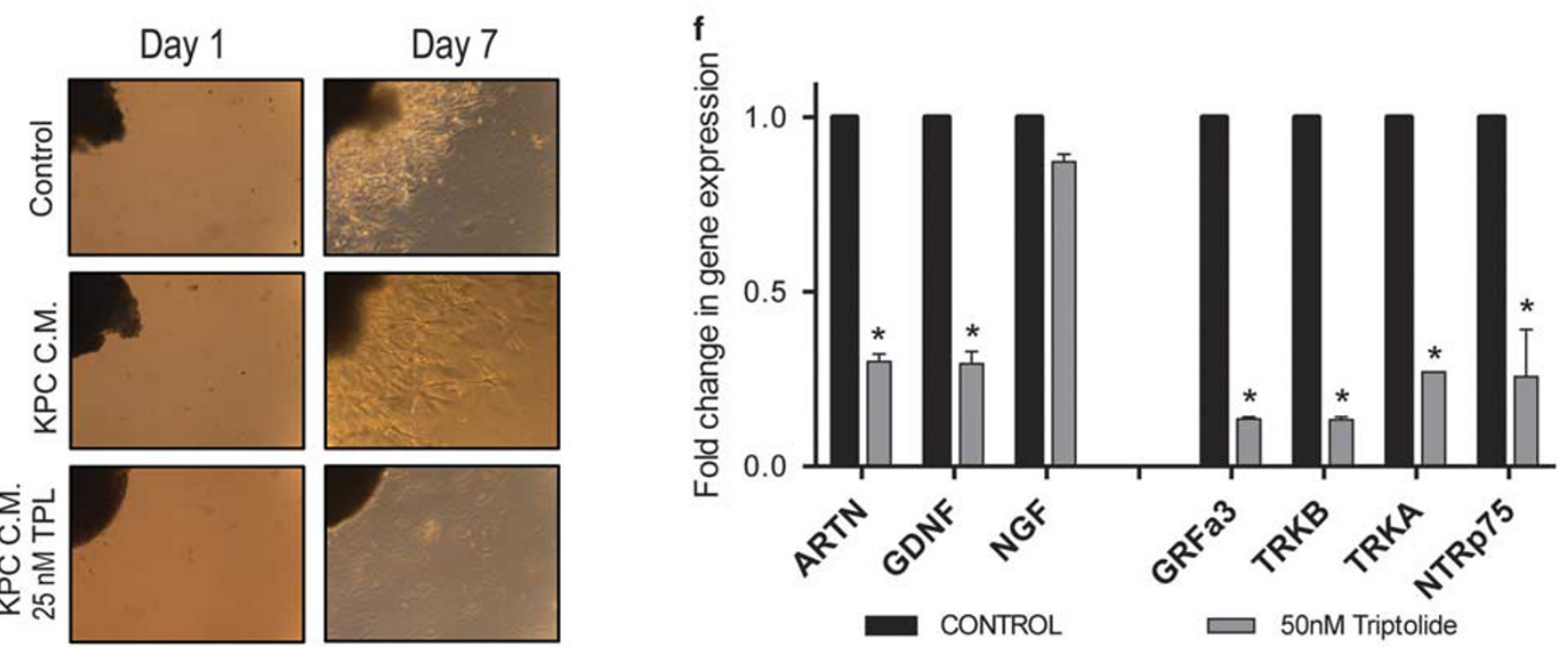
effect of NF- $\kappa$ B inhibition on tumor-neural cross talk and invasion of both tumor and neural cells.

Triptolide treatment decreased MIA PaCa-2 NGF stimulated in vitro invasion (Figure 5a), in which in vitro invasion increased from $4.180( \pm 0.737)$ invaded cells to 17.21 ( \pm 2.32) invaded cells upon NGF stimulation. Treatment with triptolide inhibited NGF stimulation and invasion decreased to $5.571( \pm 0.286)$ invaded cells, similar to control. Inhibition of in vitro invasion was also observed upon co-culture with neuroblastoma cell line, SHSY-5Y (Figure 5b). MIA PaCa-2 cells invaded significantly more when neural cells were cultured at the bottom of the well $(41.50 \pm 5.50$ invaded cells, compared with control $(9.857 \pm 2.00))$, which was inhibited by triptolide treatment $(7.00 \pm 1.00)$. Conversely, when the invasiveness of SHSY-5Y cells were tested with MIA PaCa-2 cells cultured at the bottom of the well, their increased invasiveness $(45.07 \pm 5.36$ invaded cells, compared with control (0.286 invaded cells \pm 1.43$)$ ), again, inhibited by triptolide treatment (7.00 invaded cells \pm 1.00 ; Figure $5 \mathrm{c}$ ).

Co-culture of SHSY-5Y cells increased gene expression of neurotrophins and EMT markers in MIA PaCa-2 cells (Figure 5d). Upon stimulation by neural cells, increased gene expression of artemin (1.470-fold \pm 0.349 ), glial derived neurotropic factor (GDNF; 2.153-fold \pm 0.579 ), and neural growth factor (NGF; 2.930-fold \pm 0.378 ). This also increased EMT-associated gene expression: SNAI1 (2.098-fold \pm 0.295$)$, SLUG (3.229-fold \pm 1.005$)$, TWIST1 (6.575-fold \pm 3.264$)$, ZEB1 (2.278-fold \pm 0.347$),$ CDH2 (2.722-fold \pm 0.901$)$, VIM (2.961-fold \pm 1.338 ), and MMP7 (2.206-fold \pm 0.279 ).

As an ex vivo method, murine DRGs were assayed for outgrowth when stimulated with conditioned cancer cell medium. Conditioned medium from KPC cells (KPC C.M.) increased outgrowth length of DRGs, as compared with control medium. Conditioned KPC medium with a low concentration of triptolide inhibited outgrowth completely (Figure 5e). Triptolide treatment of DRGs decreased gene expression of neurotrophins and neurotrophin receptors (Figure 5f): ARTN (0.300-fold \pm 0.022$)$, GDNF (0.294-fold \pm 0.035$), \quad$ NGF $\quad(0.873$-fold \pm 0.022$), \quad$ GRFa3 (0.135-fold \pm 0.006$), \quad$ TRKB $\quad(0.133$-fold \pm 0.009$), \quad$ TRKA (0.271-fold \pm 0.000$),$ NTRp75 (0.257-fold \pm 0.135$)$.

\section{Inhibition of Neurotrophic Signaling in vivo Decreases Neural Invasion}

Using the spontaneous pancreatic cancer murine model, KPC $\left(\mathrm{LSL}-\mathrm{Kras}^{\mathrm{G} 12 \mathrm{D} /+} ; \mathrm{LSL}-\mathrm{Trp} 53^{\mathrm{R} 172 \mathrm{H} /+} ; \mathrm{Pdx}-1-\mathrm{Cre}\right.$ transgenic), mice were treated with Minnelide, a pro-drug, water soluble formulation of triptolide. Minnelide treatment decreased expression of neurotrophins ARTN (0.456-fold \pm 0.197$)$ and GDNF $(0.167$-fold \pm 0.045$)$ and neurotrophin receptors GRFa3 $(0.085$-fold \pm 0.051$)$, TRKB $(0.038$-fold \pm 0.018$)$, TRKA $\quad(0.097$-fold \pm 0.035$), \quad$ NTRp75 $\quad(0.244$-fold \pm 0.133 ; Figure 6a). PGP 9.5 staining of the tumors revealed that the thickness and density of intratumoral nerves was decreased (Figure 6b).
Next, using an in vivo assay of invasion, MIA PaCa-2 cells were injected into the sciatic nerve of athymic mice. Treatment with Minnelide inhibited invasion of cells through the nerve, preserving nerve function. Nerve function was determined by paw spread (Figure 6c), where at day 0 both saline and Minnelide groups had a paw spread of $10.10 \pm 0.18$ and 0.23 , respectively. By day 46 , paw spread decreased to $4.40 \pm 0.27$, but in the Minnelide group only decreased to $7.40 \pm 0.52$. To examine the extent of neural invasion, tumor volume and proximal nerve diameter were measured. Tumor volume decreased from saline control $\left(65.49 \mathrm{~mm}^{3} \pm 15.62\right)$ to $0.0 \mathrm{~mm}^{3} \pm 0.0$ in Minnelide treatment group (Figure $6 \mathrm{~d}$ ). To determine the invasion within the nerve, the diameter of the nerve $2 \mathrm{~cm}$ from the primary tumor site was measured, which demonstrated a significant decrease from $1.21 \mathrm{~mm} \pm 0.23$ in saline control to $0.50 \mathrm{~mm} \pm 0.03$ in Minnelide treatment group (Figure 6e).

Taken together, these data indicate that Minnelide treatment in vivo decreases neurotrophin signaling, nerve density and size, and neural invasion.

\section{DISCUSSION}

The pancreatic tumor microenvironment contains several cellular components, which support tumor growth and progression. The interaction between cancer cells and intratumoral nerves has been studied in pancreatic cancer for several years, upon the observation that up to $100 \%$ of patients exhibit perineural invasion. Cancer cells have been known to upregulate receptors for growth factors not normally found on epithelial cells to utilize those factors secreted by other cell types in the tumor microenvironment. Pancreatic cancer cells upregulate many neural related receptors (NGFR, TRKA/B) and also secrete these neurotrophic factors themselves, providing growth factors for nerves and the capacity to benefit from the neurotrophins secreted from intratumoral nerves.

Pancreatic cancer neurotrophin stimulation by NGF has been shown to increase invasion in pancreatic cancer. ${ }^{11,12}$ In this study, we show that NF- $\kappa$ B is an essential pathway for the regulation of invasion and metastasis in pancreatic cancer and the inhibition of this pathway leads to decreased EMT related marker expression.

Although, recent evidence has come out that induction of the EMT program is not required for invasion and metastasis. ${ }^{26}$ In this study, we do demonstrate that many of these mesenchymal markers are upregulated upon the stimulation by neural cells, leading to increased invasiveness. We also see that the inhibition of NF- $\kappa$ B signaling either by triptolide/Minnelide treatment or pharmacological inhibition of NF- $\kappa \mathrm{B}$ (BAY 11-7085) decrease in vitro invasion and in vivo metastasis; decreasing EMT transcription factions, mesenchymal markers, and increasing E-Cadherin at cellular junctions.

We also show in this study that Minnelide treatment decreases tumor-nerve crosstalk through the downregulation 


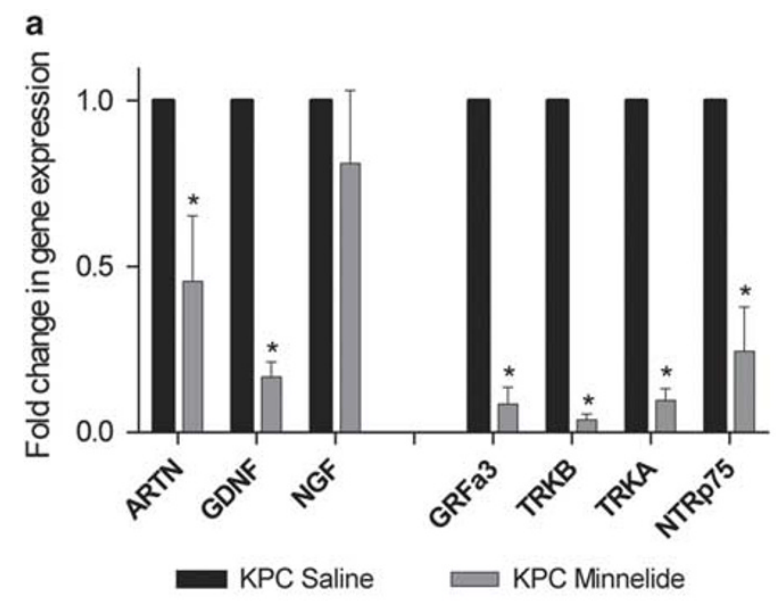

b
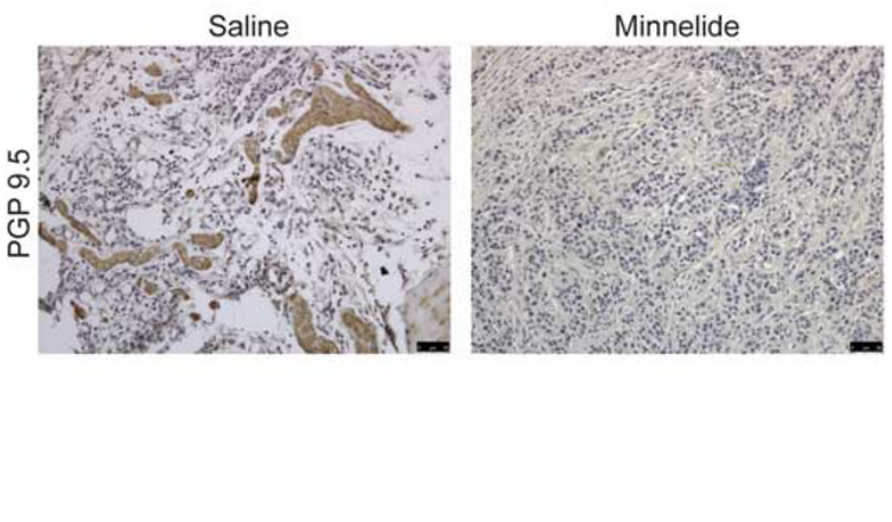

c

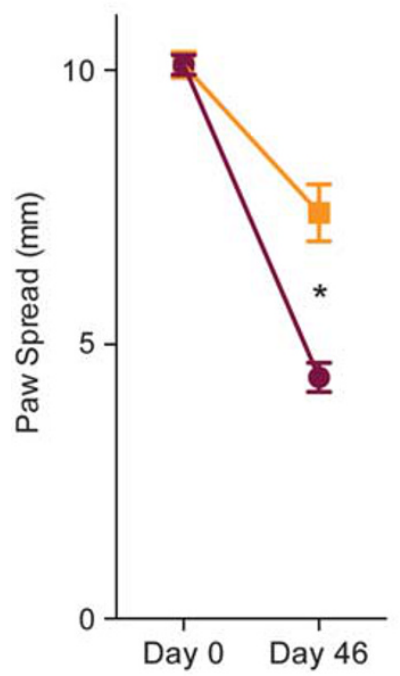

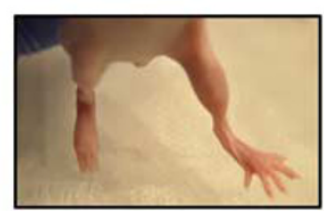

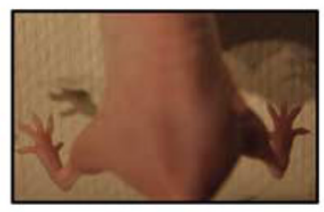

- Saline

- Minnelide d

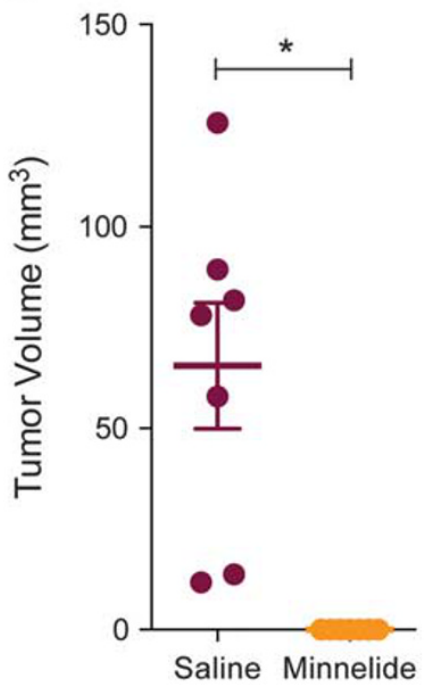

e

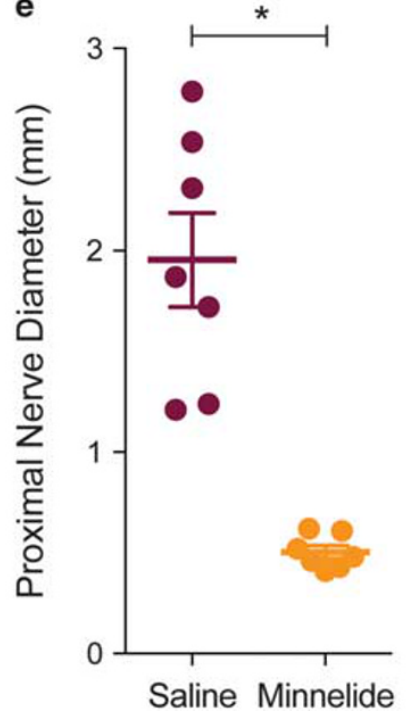

Figure 6 Inhibition of neurotrophic signaling in vivo decreases neural invasion: Minnelide (a) decreased neurotrophin and neurotrophin receptor gene expression in KPC tumors and (b) nerve size and density. In a sciatic nerve invasion model Minnelide prevented nerve destruction as measured by (c) increased paw spread as compared with saline control; (d) decreased primary tumor volume in the sciatic nerve and (e) decreased invasion through the nerve as determined by nerve diameter proximal to the primary tumor site. Each bar is representative of three or more independent experiments; error bars are represented in s.e.m.; and the asterisk $\left(^{*}\right)$ indicates a $P$-value $<0.05$.

of both neurotrophins and neurotrophin receptors within neural, as well as, cancer cells. We also demonstrate that neural outgrowth and cancer cell invasion within the nerve is inhibited by treatment with Minnelide.

Neural invasion in pancreatic cancer has been shown especially relevant in pancreatic cancer, revealing an important, but less studied mechanism of metastasis. Perineural invasion is characterized as the invasion of tumor cells into, around, and through the nerve and has been hypothesized to be responsible for local tumor recurrence upon curative resection. We demonstrate in this study that Minnelide treatment is effective in limiting neural growth within the tumor and tumor growth within the nerve. Our group has also previously shown that Minnelide is capable of preventing tumor recurrence, even upon discontinuation of therapy, indicating that this therapy may be effective in eliminating primary tumor burden, as well as, either preventing neural invasion or treating any cancer cells that have already invaded the nerves. Minnelide is currently undergoing phase I clinical trials at several sites in the United States.

Several studies have described an increase in nerve size and density within pancreatic ductal adenocarcinoma. ${ }^{7,8}$ Similarly, in this study, we saw increased nerve diameter and density in the KPC tumor, when compared to normal pancreas and we demonstrate a significant decrease in intratumoral neuronal size and density with the treatment of Minnelide.

In conclusion, this study demonstrates the importance of $\mathrm{NF}-\kappa \mathrm{B}$ activity in the induction of epithelial-mesenchymal transition, invasion, and metastasis as well as in neural invasion in pancreatic cancer.

Supplementary Information accompanies the paper on the Laboratory Investigation website (http://www.laboratoryinvestigation.org) 


\section{ACKNOWLEDGMENTS}

This study was funded by NIH grants R01-CA170946 and R01-CA124723 (to AKS); NIH grant R01-CA184274 (to SB); Katherine and Robert Goodale foundation support (to AKS), Minneamrita Therapeutics LLC (to AKS) and Alice Nomura was supported by T32 DA007097-32.

\section{DISCLOSURE/CONFLICT OF INTEREST}

University of Minnesota has a patent for Minnelide, which has been licensed to Minneamrita Therapeutics, LLC. AKS is the co-founder and the Chief Scientific Officer of this company. SB is a consultant with Minneamrita Therapeutics LLC and this relationship is managed by University of Miami. The remaining authors declare no conflict of interest.

1. Rahib L, Smith BD, Aizenberg R, et al. Projecting cancer incidence and deaths to 2030: the unexpected burden of thyroid, liver, and pancreas cancers in the United States. Cancer Res. 2014;74:2913-21.

2. Siegel RL, Miller KD, Jemal A. Cancer statistics, 2016. CA Cancer J Clin. 2016;66:7-30.

3. Rhim AD, Mirek ET, Aiello NM, et al. EMT and dissemination precede pancreatic tumor formation. Cell 2012;148:349-61.

4. Liebig C, Ayala G, Wilks JA, et al. Perineural invasion in cancer: a review of the literature. Cancer 2009;115:3379-91.

5. Liu B, Lu KY. Neural invasion in pancreatic carcinoma. Hepatobiliary Pancreat Dis Int 2002;1:469-76.

6. Pour PM, Bell RH, Batra SK. Neural invasion in the staging of pancreatic cancer. Pancreas 2003;26:322-5.

7. Ceyhan GO, Bergmann F, Kadihasanoglu M, et al. Pancreatic neuropathy and neuropathic pain-a comprehensive pathomorphological study of 546 cases. Gastroenterology 2009;136:177-86 e1.

8. Demir IE, Ceyhan GO, Rauch U, et al. The microenvironment in chronic pancreatitis and pancreatic cancer induces neuronal plasticity. Neurogastroenterol Motil. 2010;22:480-90 e112-113.

9. Ceyhan GO, Giese NA, Erkan M, et al. The neurotrophic factor artemin promotes pancreatic cancer invasion. Ann Surg 2006;244:274-81.

10. Okada Y, Eibl G, Duffy JP, et al. Glial cell-derived neurotrophic factor upregulates the expression and activation of matrix metalloproteinase9 in human pancreatic cancer. Surgery 2003;134:293-9.

11. Okada Y, Eibl G, Guha S, et al. Nerve growth factor stimulates MMP-2 expression and activity and increases invasion by human pancreatic cancer cells. Clin Exp Metastasis 2004;21:285-92.

12. Zhu Z, Kleeff J, Kayed $\mathrm{H}$, et al. Nerve growth factor and enhancement of proliferation, invasion, and tumorigenicity of pancreatic cancer cells. Mol Carcinog 2002;35:138-47.
13. Helbig G, Christopherson 2nd KW, Bhat-Nakshatri P, et al. NF-kappaB promotes breast cancer cell migration and metastasis by inducing the expression of the chemokine receptor CXCR4. J Biol Chem 2003;278: 21631-8.

14. Maier $\mathrm{HJ}$, Schmidt-Strassburger $\mathrm{U}$, Huber MA, et al. NF-kappaB promotes epithelial-mesenchymal transition, migration and invasion of pancreatic carcinoma cells. Cancer Lett 2010;295:214-28.

15. Fujioka S, Sclabas GM, Schmidt C, et al. Inhibition of constitutive NF-kappa B activity by I kappa B alpha M suppresses tumorigenesis. Oncogene 2003;22:1365-70.

16. Wang W, Abbruzzese JL, Evans DB, et al. The nuclear factor-kappa B RelA transcription factor is constitutively activated in human pancreatic adenocarcinoma cells. Clin Cancer Res 1999;5:119-27.

17. Alsaied OA, Sangwan V, Banerjee S, et al. Sorafenib and triptolide as combination therapy for hepatocellular carcinoma. Surgery 2014;156: 270-9.

18. Banerjee S, Sangwan V, McGinn O, et al. Triptolide-induced cell death in pancreatic cancer is mediated by O-GICNAC modification of transcription factor Sp1. J Biol Chem. 2013;288:33927-38.

19. Rousalova I, Banerjee S, Sangwan V, et al. Minnelide: a novel therapeutic that promotes apoptosis in non-small cell lung carcinoma in vivo. PLoS One 2013;8:e77411.

20. Banerjee S, Nomura A, Sangwan V, et al. Minnelide reduces CD133+ tumors initiating "stem-like" cells in a syngenic murine model of pancreatic ductal adenocarcinoma. Clin Cancer Res 2014;20: 2388-99.

21. Reichert M, Takano S, Heeg S, et al. Isolation, culture and genetic manipulation of mouse pancreatic ductal cells. Nat Protoc. 2013;8: 1354-65.

22. Chua HL, Bhat-Nakshatri $\mathrm{P}$, Clare $\mathrm{SE}$, et al. NF-kappaB represses E-cadherin expression and enhances epithelial to mesenchymal transition of mammary epithelial cells: potential involvement of ZEB-1 and ZEB-2. Oncogene 2007;26:711-24.

23. Huber MA, Azoitei N, Baumann B, et al. NF-kappaB is essential for epithelial-mesenchymal transition and metastasis in a model of breast cancer progression. J Clin Invest 2004;114:569-81.

24. Maier HJ, Schmidt-Strassburger U, Huber MA, et al. NF-kappaB promotes epithelial-mesenchymal transition, migration and invasion of pancreatic carcinoma cells. Cancer Lett 2010;295:214-28.

25. Chugh R, Sangwan V, Patil SP, et al. A preclinical evaluation of minnelide as a therapeutic agent against pancreatic cancer. Sci Transl Med 2012;4:156ra39.

26. Zheng X, Carstens JL, Kim J, et al. Epithelial-to-mesenchymal transition is dispensable for metastasis but induces chemoresistance in pancreatic cancer. Nature 2015;527:525-30. 